\title{
Absence of true long-range orbital order in a two-leg Kondo ladder
}

\author{
J. C. Xavier ${ }^{1}$ and A. L. Malvezzi ${ }^{2}$ \\ ${ }^{1}$ Instituto de Física, Universidade Federal de Uberlândia, Caixa Postal 593, 38400-902 Uberlândia, MG, Brazil \\ ${ }^{2}$ Departamento de Física, Faculdade de Ciências, Universidade Estadual Paulista, Caixa Postal 473, 17015-970 Bauru, SP, Brazil
}

(Received 3 August 2006; revised manuscript received 11 June 2007; published 16 July 2007)

\begin{abstract}
We investigate, through the density-matrix renormalization group and the Lanczos technique, the possibility of a two-leg Kondo ladder presenting an incommensurate orbital order. Our results indicate staggered shortrange orbital order at half-filling. Away from half-filling our data are consistent with incommensurate quasilong-range orbital order. We also observed that an interaction between the localized spins enhances the rungrung current correlations.
\end{abstract}

DOI: 10.1103/PhysRevB.76.014422

\section{INTRODUCTION}

In 1985, it was observed that the heavy fermion superconductor $\mathrm{URu}_{2} \mathrm{Si}_{2}$ presents a second-order phase transition at $17.5 \mathrm{~K} .{ }^{1}$ This phase transition is characterized by sharp features in the specific heat ${ }^{1}$ and several other thermodynamic properties (see, e.g, Ref. 2 and references therein). From the Landau theory, it is expected an ordered moment, due to the large specific heat jump (entropy) in this transition, to be about $m \sim 0.5 \mu_{B} \cdot{ }^{3}$ However, the size of the staggered moment measured by neutron scattering measurements is $m$ $\sim 0.03 \mu_{B}{ }^{1}$. The order parameter associated with this phase transition is, at the present moment, not established, and it is a challenge to discover the nature of the hidden order behind the transition.

Many theoretical groups have proposed several kinds of hidden order. ${ }^{2,4-8}$ But until now, experiments were not able to establish which is the correct one. Certainly, also from the theoretical point of view, more studies are needed to clarify the correct order associated with this mysterious phase transition. In this front, we present here a numerical study of a microscopic model for heavy fermion systems.

In this work we focus on the order parameter proposed a few years ago by Chandra and collaborators. ${ }^{2}$ They suggested the existence of a hidden incommensurate orbital order in the heavy fermion $\mathrm{URu}_{2} \mathrm{Si}_{2}$ below the second-order phase transition. The orbital order phase is associated with currents circulating around the plaquettes, as illustrated in Fig. 1. In the case of $\mathrm{URu}_{2} \mathrm{Si}_{2}$, this currents produce a very weak orbital moment of $0.02 \mu_{B}$ that explains the large entropy loss. ${ }^{2}$

Very recently, neutron scattering measurements were unable to detect the orbital order in the heavy fermion $\mathrm{URu}_{2} \mathrm{Si}_{2} \cdot{ }^{9}$ Although the orbital order was not detected, it is not possible yet to discard it as the hidden order due to the resolution limitation of the experiments performed. Note that the orbital order signature in the neutron scattering experiments is expected to be 50 times weaker than the spin order one at ambient pressure. ${ }^{10}$

Our goal in this work is to investigate the existence of an incommensurate orbital order in the Kondo lattice model (KLM). This model is the simplest one believed to present the physics of heavy fermions materials ${ }^{11}$ (see next section). Our approach will be numerical, through the density-matrix renormalization group ${ }^{12}$ (DMRG) and the Lanczos technique. ${ }^{13}$ These techniques are nonperturbative, however
PACS number(s): 75.10.-b, 71.10.Pm, 75.30.Mb

limited by the system size. For this reason, we consider the two-leg Kondo ladder (2-LKL), which is the simplest geometry able to present an orbital order.

The orbital order, also called the flux or orbital current phase, has already been discussed in the context of the hightemperature superconductors. The standard two-leg $t$ - $J$ ladder model presents a short-range orbital order, ${ }^{14}$ while an extended version has long-range orbital order for some parameters. ${ }^{15}$ A recent detailed discussion of the orbital order in the context of a Hubbard model can be found in Ref. 16.

We close this section mentioning that a model very similar to the KLM was used to describe the magnetism of $\mathrm{URu}_{2} \mathrm{Si}_{2}$. Sikkema and collaborators, ${ }^{17}$ through a mean-field calculation, showed that the Ising-Kondo lattice model with transverse field presents a weak ordered moment, similar to the one observed in experiments. However, the Ising-KLM model was not able to reproduce the large specific heat jump.

\section{MODEL}

In order to investigate the heavy fermion systems the minimum ingredients that a microscopic model must consider are two types of electrons: the conduction electrons in the $s, p$, or $d$, orbital as well the electrons in the inner $f$ orbitals. ${ }^{18}$ In the literature there are two well-known standard models that consider these two kind of electrons: the periodic Anderson model (PAM) and the KLM. ${ }^{18}$ In an appropriate parameter regime [mainly (i) the mobility of the $f$ electrons is very small, which is relevant for the heavy fermion system, and (ii) that the Coulomb interaction of the electrons in the $f$ orbitals is very large] Schrieffer and Wolff ${ }^{18,19}$ showed that the KLM can be derived from the PAM. We consider in this work the KLM which has fewer degrees of freedom per unit cell than the PAM and it is easier to explore numerically.

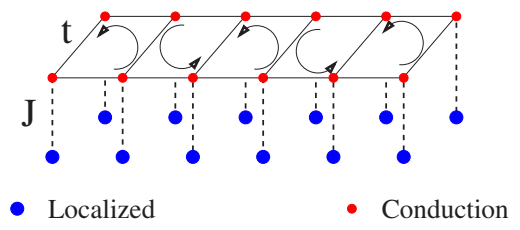

FIG. 1. (Color online) A schematic representation of the two-leg Kondo ladder. It also shows the circulating currents around the plaquettes (in this example a staggered one). 
The KLM incorporates an interaction between the localized spins and the conduction electrons via an exchange interaction $J$. To attack this model in two or three dimensions by unbiased nonperturbative numerical approaches is an impossible task at the present moment. However, it is possible to consider quasi-one-dimensional systems such as the $N$-leg ladder model.

We consider the 2 -LKL with $2 \times L$ sites defined by

$$
H_{K M}=-\sum_{\langle i, j\rangle, \sigma}\left(c_{i, \sigma}^{\dagger} c_{j, \sigma}+\text { H.c. }\right)+J \sum_{j} \mathbf{S}_{j} \cdot \mathbf{s}_{j}+J_{A F} \sum_{\langle i, j\rangle} \mathbf{S}_{i} \cdot \mathbf{S}_{j},
$$

where $c_{j \sigma}$ annihilates a conduction electron in site $j$ with spin projection $\sigma, \mathbf{S}_{j}$ is a localized spin $\frac{1}{2}$ operator, $\mathbf{s}_{j}$ $=\frac{1}{2} \Sigma_{\alpha \beta} c_{j, \alpha}^{\dagger} \sigma_{\alpha \beta} c_{j, \beta}$ is the conduction electron spin density operator, and $\sigma_{\alpha \beta}$ are Pauli matrices. Here $\langle i j\rangle$ denote nearestneighbor sites, $J>0$ [when the KLM is deduced from the PAM one obtains $J>0$ (Ref. 18)] is the Kondo coupling constant between the conduction electrons and the local moments, and the hopping amplitude was set to unity to fix the energy scale.

We also consider an interaction between the localized spins $J_{A F}$; we choose $J_{A F}>0$ since antiferromagnetism was observed in $\mathrm{URu}_{2} \mathrm{Si}_{2} .{ }^{20}$ The same model above also represents the manganites when $J<0 .{ }^{21}$ In this latter case, the interaction between the localized spins seems to be important to stabilize some phases. ${ }^{21}$ This is motivation to also consider this interaction. Note that several others terms in the Hamiltonian could also be included, like the Coulomb interaction of the electrons in the conduction band, extra electrons hopping, etc. However, at the present moment, there is no evidence indicating that such extra terms are relevant to the low-energy physics of the heavy fermion systems. Up to now, it has been well established that $J$ is essential to describe the magnetism observed in the heavy fermion systems. At small values of $J$, an antiferromagnetic long-range order (LRO) is expected due the Ruderman-Kittel-Kasuya-Yosida interaction, whereas for large $J$ a paramagnetic phase emerges. Doniach ${ }^{22}$ was the first to point out the existence of a quantum critical point (QCP) due the competition between these two phases.

Unlike other models, such as the $t-J$ model, much less is known about the Kondo lattice model, even in the onedimensional version, where the ground state of the Kondo chain is quite well known ${ }^{18}$ (see also Ref. 23). New phases have been reported recently, such as a ferromagnetic phase ${ }^{24}$ inserted into the paramagnetic phase as well as a dimerized phase at quarter-filling. ${ }^{25}$ The latter has been questioned recently by Hotta and Shibata. ${ }^{26}$ Those authors claim that the dimerized phase is an artifact of the open boundary conditions. Indeed, the boundary condition is very important, as well as the number of sites considered. In Ref. 26 the authors observed, mainly, that with an odd number the sites the dimer state does not exist. The parity of the number of sites is thus very relevant and an odd number destroys the dimerization. ${ }^{27}$

In quasi-one-dimensional systems, such as the $N$-leg ladders, very few nonperturbative studies have been reported.
Recently, quantum Monte Carlo ${ }^{28}$ (QMC) and $\mathrm{DMRG}^{29}$ calculations of the half-filled Kondo lattice model in small clusters found the existence of a QCP at $J \sim 1.45$, in agreement with previous approximate approaches ${ }^{30,31}$ (see also Ref. 32). Note that the QMC calculations were feasible only at halffilling, where the famous sign problem is absent. Moreover, the DMRG results of the $N$-LKL at half-filling show that the spin and charge gaps are nonzero for any number of legs and Kondo coupling $J$. These results are quite different from the well known $N$-leg Heisenberg ladders where the spin gap is zero for an even number of legs. ${ }^{33}$

The phase diagram of the 2-LKL has also been explored numerically. ${ }^{34}$ In this case, a ferromagnetic phase was observed only for small densities, very distinctively from the phase diagram of the one-dimensional KLM, where the ferromagnetism is present at all electronic densities for large values of $J$. However, it is similar to the mean-field phase diagram of the tridimensional KLM. ${ }^{35}$ In this sense, the 2-LKL presents a better signature of the phases appearing in real systems than its one-dimensional version. It is interesting that dimerization was also observed in the $2-\mathrm{LKL}^{34}$ at conduction electron densities $n=1 / 4$ and $n=1 / 2$. As in the one-dimensional version, the RKKY interaction explains these unusual spin structures. In fact, in some real heavy fermion systems some unusual spin order structures have indeed been observed. ${ }^{36}$

Here, we consider electronic densities $n$ larger than 0.4, where a paramagnetic phase has been observed.$^{34}$ In particular, we focus on the electronic densities $n=1$ and $n=0.8$. We choose these densities since the magnitude of the rung-rung current correlation is larger for larger electronic densities. We investigate the model with the DMRG technique under open boundary conditions and use the finite-size algorithm for sizes up to $2 \times L=120$, keeping up to $m=1600$ states per block in the final sweep. The discarded weight was typically about $10^{-5}-10^{-7}$ in the final sweep. We also cross-checked our results with the Lanczos technique for small systems.

\section{RESULTS}

Before presenting our results, we briefly discuss the order parameter associated with a circulating current phase. Such a phase breaks rotational and translational as well as timereversal symmetries. The appropriated order parameter to detect this phase is the current between two nearest-neighbor sites, i.e., $\left\langle\hat{J}_{l, j}\right\rangle$, where the current operator between two nearest neighbors $i$ and $j$ is given by

$$
\hat{J}_{l, j}=i \sum_{\sigma}\left(c_{l, \sigma}^{\dagger} c_{j, \sigma}-c_{j, \sigma}^{\dagger} c_{l, \sigma}\right),
$$

Strictly speaking, a spontaneous symmetry breaking only appears in the thermodynamic limit. Only in this limit $\left\langle\hat{J}_{l, j}\right\rangle$ $\neq 0$ in the ordered phase. The signature of a spontaneous symmetry breaking appears in the two-point correlation function of the operator that measures the symmetry. We utilize this fact to infer the orbital order. If a continuous symmetry is broken, no long-range order exist at finite temperature in one and two dimensions, as stated by the Mermin-Wagner-Hohenberg theorem. ${ }^{37}$ At zero temperature 
true long-range order is still possible in two dimensions, while in one dimension only a quasi-long-range order can manifest; i.e., the two-point correlation function decay is algebraic. However, if the translational symmetry (a discrete symmetry) is broken, even in one dimension a true longrange order may exist (a famous example is the dimerized phase of the Majumdar-Ghosh model ${ }^{38-40}$ ). Since the translational symmetry is broken in the orbital phase, true longrange order may occurs in the ground state of the 2-LKL.

In order to observe any trace of orbital order in the ground-state wave function of the 2-LKL, we measure the rung-rung current correlations defined as

$$
C(l, k)=\langle\hat{J}(l) \hat{J}(k)\rangle,
$$

where $\hat{J}(l)$ is the rung current operator for the $l$ th rung given by

$$
\hat{J}(l)=i \sum_{\sigma}\left(c_{l 2, \sigma}^{\dagger} c_{l 1, \sigma}-c_{l 1, \sigma}^{\dagger} c_{l 2, \sigma}\right)
$$

and $c_{l \lambda, \sigma}$ annihilates a conduction electron on rung $l$ and leg $\lambda=1,2$ with spins $\sigma=\uparrow, \downarrow$. Since we work with open boundary conditions, it is convenient to define an averaged rungrung current correlation in order to minimize boundary effects. We have defined the averaged rung-rung current correlation as

$$
C(l)=\frac{1}{M} \sum_{|i-k|=l}\langle\hat{J}(i) \hat{J}(k)\rangle,
$$

where $M$ is the number of site pairs $(i, k)$ satisfying $l=\mid i$ $-k \mid$. Typically, $M$ in our calculation varies from 3 to 10 .

There is true long-range orbital order if $\lim _{l \rightarrow \infty} C(l) \neq 0$. Through this criterion, we can infer the existence of the orbital order by measuring $C(l)$ at large distances. If $C(l)$ has an exponential decay, the linear-log plot shows a linear decay. On the other hand, if $C(l)$ has an power law decay, the log-log plot presents a linear decay.

We also measure the cosine transform of $C(l)$, i.e.,

$$
N(q)=\sum_{l=1}^{L} C(l) \cos (l q),
$$

in order to infer about periodicity of the oscillatory part of $C(l)$.

In the next two subsections we investigate these correlations for the 2-LKL at half-filling and close to half-filing. We did not find any evidence of long-range orbital order in the ground state of the 2-LKL. Our results show that the rungrung current correlation has an exponential decay at halffilling. Close to half-filling our results indicate an incommensurate quasi-long range orbital order.

\section{A. Half-filling}

We start presenting some results for the conduction electron density $n=1$. We observed, in this case, that the averaged rung-rung current correlation behaves as
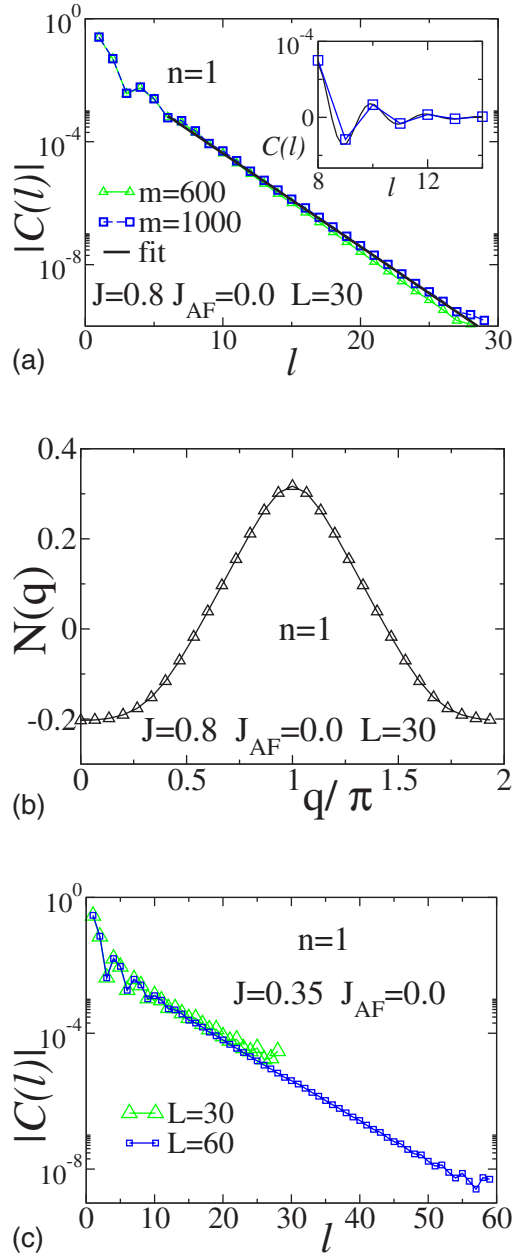

FIG. 2. (Color online) (a) The linear-log plot of $|C(l)|$ for two distinct values of $m$ with $L=30$ at half-filling. The solid line in (a) corresponds to a fit of Eq. (4) with $\xi=1.43$ and $a_{0}=0.06$; the rms percent error is 0.18 . Inset: $C(l)$ vs distance with $m=1000$. Only a few sites are presented. The couplings are $J=0.8$ and $J_{A F}=0$. (b) The cosine transform $N(q)$ of $C(l)$ presented in Fig. 1(a) with $m$ $=1000$. (c) The linear-log plot of $|C(l)|$ for two distinct size, both with $m=1000$. The couplings are $J=0.35$ and $J_{A F}=0$.

$$
C(l)=a_{0}(-1)^{l} \exp (-l / \xi)
$$

for all values of $J$ and $J_{A F}$ explored in this work. In Fig. 2(a), we present a typical example of the magnitude of $C(l)$ at half-filling for a system size $L=30$. As we see, our results indicate strongly that $C(l)$ has an exponential decay due to the linear decay in the linear-log plot. The inset in Fig. 2(a) also shows that $C(l)$ is staggered. The solid line in Fig. 2(a) corresponds to a fit of Eq. (4) with $a_{0}=0.06$ and a decay length $\xi=1.43 .{ }^{41}$ We performed a least-squares fitting, resulting in a root mean square (rms) of 0.0018 and a correlation coefficient of 0.996 . We found that $C(l)$ has a very small dependence on the number $m$ of states retained in the truncation process for $J>0.8$, as can be observed in Fig. 2(a). It is very hard to get accurate results for $J<0.8$, however, even for small values of $J$. We believe we have captured the correct qualitative behavior. Nevertheless, we present most of our results for $J>0.8$, where the results are more accurate. 

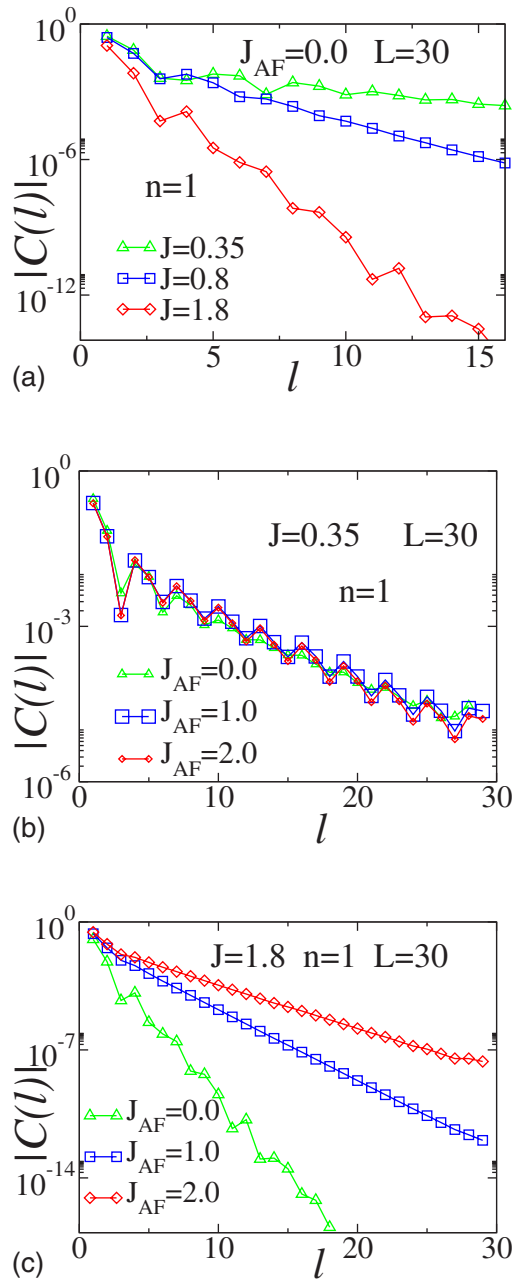

FIG. 3. (Color online) The linear-log plot of $|C(l)|$ for a set of representative values of $J$ and $J_{A F}$ for $L=30$ at half-filling. (a) $|C(l)|$ for $J_{A F}=0$ and $J=0.35,0.8$ and 1.8. (b) $|C(l)|$ for $J=0.35$ and some values of $J_{A F}$. (c) Same as (b) but for $J=1.8$.

The signature of the sign alternation is observed through the cosine transform of $C(l)$. In Fig. 2(b), we show the cosine transform of $C(l)$ presented in Fig. 2(a) with $m=1000$. Clearly, we observe a peak at $q=\pi$ due the sign alternation of $C(l)$. Note that the finite-size effects are small, as can be seen in Fig. 2(c). For this reason, we restrict most of our calculations to system size $2 \times 30$ in order to save computational time.

Our results indicate that for small $J$, where the RKKY is expected to be dominant, the rung-rung current correlations have a larger correlation length as we see in Fig. 3(a). On the other hand, for large $J$, which favors formation of singles, the correlation length is smaller. This result is expected, since for $J \rightarrow \infty$ the rung-rung current correlations must go to zero.

The Hamiltonian shown in Eq. (1) with $J_{A F}=0$ does not present a long-range orbital order at half-filling, as we have observed. Since $J_{A F}$ seems to be important to stabilize some phases for $J<0,{ }^{21}$ it may be possible that it also stabilizes the orbital phase for $J>0$. For these reasons, we also investigate the effect of $J_{A F}$ in the ground state of the 2-LKL. As we see in Fig. 3(b), for small values of $J, J_{A F}$ does not affect significantly $C(l)$. On the other hand, for larger $J$ as shown in

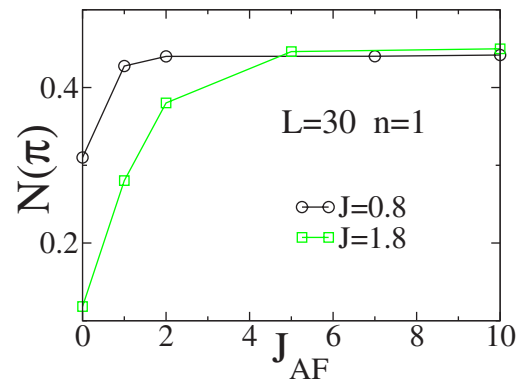

FIG. 4. (Color online) The peak intensity of $N(q)$ at $q=\pi$ for $J=0.8$ and $J=1.8$ as a function of $J_{A F}$ for a system size $L=30$ at half-filling.

Fig. 3(c), $J_{A F}$ clearly enhances the correlation length. Although $J_{A F}$ enhanced $C(l)$, at half-filling only short-range orbital order is observed for several parameters investigated.

At half-filling, for all parameters studied, $N(q)$ always presents a peak at $q=\pi$. In Fig. 4, we present this peak intensity for $J=0.8$ and $J=1.8$ as a function of $J_{A F}$. As we see, the peak intensity increases with $J_{A F}$ and saturates for large $J_{A F}$ around $\sim 0.45$.

Our main conclusion, for the half-filling case, is the absence of long-range orbital order. Note that it may be possible that the inclusion of the Coulomb interaction between the electrons in the conduction band leads the system into a phase with long-range orbital order, as occurs in an extend $t-J$ model. ${ }^{15}$ This is under investigation at the present moment by one of the authors.

\section{B. Close to half-filling}

Away from half-filling the DMRG calculation of $C(l)$ is less stable; for this reason, we consider system sizes smaller than $2 \times 40$ and keep up to $m=1600$ states in the truncation process. Although we obtained results for a couple of densities away from half-filling, we focus on the density $n=0.8$ where the magnitude of $C(l)$ is larger. For small densities it is very hard to get accurate results since the current intensity is very small. In Fig. 5(a), we present the log-log plot of $|C(l)|$ at conduction electron density $n=0.8$ for a system size $2 \times 30$ with $J=0.8$ and $J_{A F}=1.0$ for two different values of $m$. Since in the log-log plot we obtain a linear decay (see the solid line in this figure), $C(l)$ must have a power law decay. If we use a linear-log plot, our data do not have a linear decay. As can be seen from Fig. 5, it is very hard to get good accuracy even working with $m=1600$ states. Although we were not able to obtain the current-current correlations at large distances with a high accuracy, we believe we have captured the correct behavior, i.e., a power law decay. The large oscillations appearing in Fig. 5 are due to the fact that some values of $C(l)$ are very close to zero.

Since our data of $C(l)$ in the log-log plot strongly suggest a power law decay close to half-filling (note that for the half-filling case the decay is exponential), we tried to fit $C(l)$ with the function

$$
C_{f i t}(l)=a_{0} \frac{\cos (n \pi l)}{l^{\alpha_{1}}}+a_{1} \frac{\cos (2 n \pi l)}{l^{\alpha_{2}}},
$$

where $n=0.8$ is the density. The dashed curve in Fig. 5(a) corresponds to a fitting of our data with $m=1600$. We were 

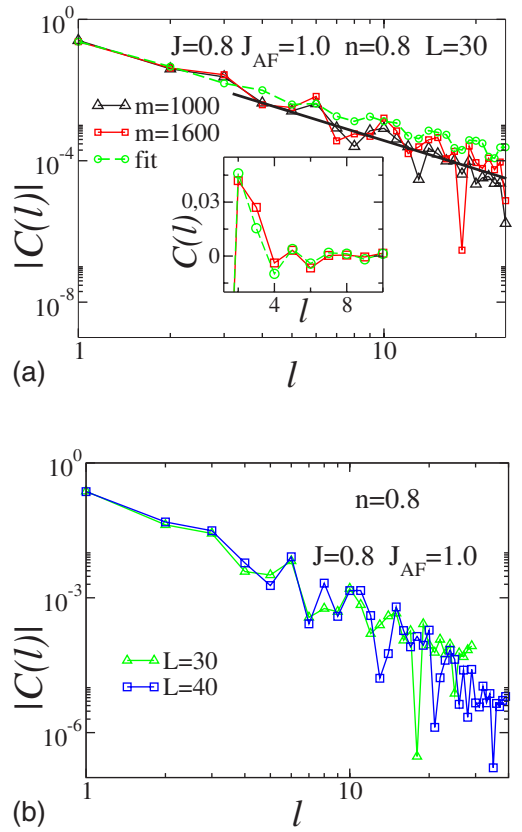

FIG. 5. (Color online) The log-log plot of $|C(l)|$ for the conduction electron density $n=0.8$. (a) $|C(l)|$ vs distance for $J=0.8, J_{A F}$ $=1.0$, and $L=30$ for two distinct values of $m$. The dashed line is the fit using Eq. (5) with $\alpha_{1}=2, \alpha_{2}=3, a_{0}=0.16$, and $a_{1}=-0.33$; the rms percent error is 6 with a correlation coefficient of 0.998 . The solid line is a guide to the eye. (b) $|C(l)|$ for the same parameters of (a) and two distinct sizes, both cases with $m=1600$. Inset: $C(l)$ vs distance with $m=1600$. Only a few sites are presented.

not able to reproduce $C(l)$ precisely; however, the general behavior is quite well described.

Note also that finite-size effects are larger away from halffiling, as we can see by comparing Figs. 5(b) and 2(c). It is important to mention that we observed, away from halffilling and in very few distances $l$, that the sign of the averaged correlation $C(l=|j-k|)$ does not has the same sign of $C(j, k)$ for some pairs of $(j, k)$ satisfying $l=|j-k|$. This does not seem to be due to the number of states kept in the truncation process since we also obtained the same effect for small clusters with exact diagonalization.

In Fig. 6 we present $N(q)$ for a representative set of parameters at conduction electron density $n=0.8$. As shown in Fig. 6, there is no peak at $q=\pi$, signaling an absence of staggered rung-rung current correlations. For the conduction

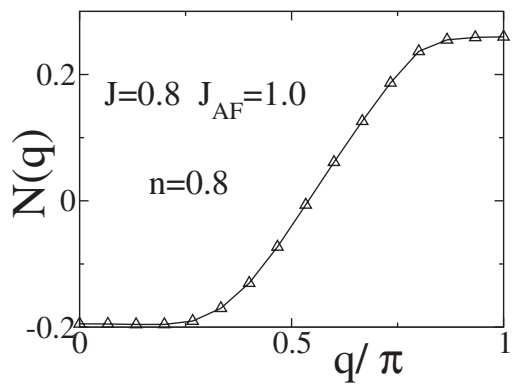

FIG. 6. The cosine transform $N(q)$ of $C(l)$ presented in Fig. 5(a) with $m=1600$.

electron density $n=0.8$ we observed a cusp at $q=n \pi$. These results indicate that close to half-filling the 2-LKL presents an incommensurate quasi-long-range orbital order.

\section{CONCLUSION}

In this paper, we have investigated the possibility of a two-leg Kondo ladder presenting an orbital order. In particular, we focus on the densities $n=1$ and $n=0.8$. For the several couplings investigated we did not find any trace of true longrange orbital order, which would be relevant to explain the large entropy loss observed in the second-order phase transition of $\mathrm{URu}_{2} \mathrm{Si}_{2}$. Our data indicate that the half-filling case presents a staggered short-range orbital order, while close to half-filling our results are consistent with an incommensurate quasi-long-range orbital order. Although we did not find evidence of a long-range orbital order in the ground state of the two-leg Kondo ladder, we cannot yet completely discard this possibility. It may occur that an extended version of the Kondo lattice model presents the long-range orbital order. So we may conclude that either the orbital phase does not exist and is not the origin of the mysterious phase transition observed in the the heavy fermion $\mathrm{URu}_{2} \mathrm{Si}_{2}$ or the standard Kondo lattice model is not able to reproduce the correct order observed in the experiments.

\section{ACKNOWLEDGMENTS}

The authors thank E. Miranda for useful discussions. This work was supported by the Brazilian agencies CNPq, FAPEMIG, and FAPESP.
${ }^{1}$ T. T. M. Palstra, A. A. Menovsky, J. van den Berg, A. J. Dirkmaat, P. H. Kes, G. J. Nieuwenhuys, and J. A. Mydosh, Phys. Rev. Lett. 55, 2727 (1985).

${ }^{2}$ P. Chandra, P. Coleman, J. A. Mydosh, and V. Tripathi, Nature (London) 417, 831 (2002).

${ }^{3}$ W. J. L. Buyers, Physica B 223, 9 (1996).

${ }^{4}$ C. M. Varma and L. Zhu, Phys. Rev. Lett. 96, 036405 (2006).

${ }^{5}$ D. F. Agterberg and M. B. Walker, Phys. Rev. B 50, 563 (1994).

${ }^{6}$ H. Ikeda and Y. Ohashi, Phys. Rev. Lett. 81, 3723 (1998).
${ }^{7}$ P. Santini and G. Amoretti, Phys. Rev. Lett. 73, 1027 (1994).

${ }^{8}$ A. Kiss and P. Fazekas, Phys. Rev. B 71, 054415 (2005).

${ }^{9}$ C. R. Wiebe, G. M. Luke, Z. Yamani, A. A. Menovsky, and W. J. L. Buyers, Phys. Rev. B 69, 132418 (2004).

${ }^{10}$ V. Tripathi, P. Chandra, and P. Coleman, J. Phys.: Condens. Matter $\mathbf{1 7}, 5285$ (2005).

${ }^{11}$ A. C. Hewson, The Kondo Problem to Heavy Fermions (Cambrige University Press, Cambridge, England, 1993).

${ }^{12}$ S. R. White, Phys. Rev. Lett. 69, 2863 (1992). 
${ }^{13}$ E. Dagotto, Rev. Mod. Phys. 66, 763 (1994).

${ }^{14}$ D. J. Scalapino, S. R. White, and I. Affleck, Phys. Rev. B 64, 100506(R) (2001).

${ }^{15}$ U. Schollwöck, S. Chakravarty, J. O. Fjærestad, J. B. Marston, and M. Troyer, Phys. Rev. Lett. 90, 186401 (2003).

${ }^{16}$ J. O. Fjærestad, J. B. Marston, and U. Schollwöck, Ann. Phys. (N.Y.) 321, 894 (2006).

${ }^{17}$ A. E. Sikkema, W. J. L. Buyers, I. Affleck, and J. Gan, Phys. Rev. B 54, 9322 (1996).

${ }^{18}$ H. Tsunetsugu, M. Sigrist, and K. Ueda, Rev. Mod. Phys. 69, 809 (1997)

${ }^{19}$ J. R. Schrieffer and P. A. Wolff, Phys. Rev. 149, 491 (1966).

${ }^{20}$ C. Broholm, J. K. Kjems, W. J. L. Buyers, P. Matthews, T. T. M. Palstra, A. A. Menovsky, and J. A. Mydosh, Phys. Rev. Lett. 58, 1467 (1987).

${ }^{21}$ E. Dagotto, Nanoscale Phase Separation and Colossal Magnetoresistance (Springer-Verlag, Berlin, 2002).

${ }^{22}$ S. Doniach, Physica B \& C 91B, 231 (1977).

${ }^{23}$ M. Gulacsi, Adv. Phys. 53, 769 (2004).

${ }^{24}$ I. P. McCulloch, A. Juozapavicius, A. Rosengren, and M. Gulacsi, Phys. Rev. B 65, 052410 (2002).

${ }^{25}$ J. C. Xavier, R. G. Pereira, E. Miranda, and I. Affleck, Phys. Rev. Lett. 90, 247204 (2003).

${ }^{26}$ C. Hotta and N. Shibata, Physica B 378-380, 1039 (2006).

${ }^{27}$ J. C. Xavier and E. Miranda (unpublished).
${ }^{28}$ F. F. Assaad, Phys. Rev. Lett. 83, 796 (1999).

${ }^{29}$ J. C. Xavier, Phys. Rev. B 68, 134422 (2003).

${ }^{30}$ Z. P. Shi, R. R. P. Singh, M. P. Gelfand, and Z. Q. Wang, Phys. Rev. B 51, 15630 (1995).

${ }^{31}$ Z. Wang, X. P. Li, and D. H. Lee, Physica B 199-200, 463 (1994).

${ }^{32}$ K. S. Beach, P. A. Lee, and P. Monthoux, arXiv:cond-mat/030633 (unpublished).

${ }^{33}$ E. Dagotto and T. M. Rice, Science 271, 618 (1996).

${ }^{34}$ J. C. Xavier, E. Miranda, and E. Dagotto, Phys. Rev. B 70, 172415 (2004).

${ }^{35}$ C. Lacroix and M. Cyrot, Phys. Rev. B 20, 1969 (1979).

${ }^{36}$ E. Granado, P. G. Pagliuso, C. Giles, R. Lora-Serrano, F. Yokaichiya, and J. L. Sarrao, Phys. Rev. B 69, 144411 (2004).

${ }^{37}$ A. Auerbach, Interacting Electrons and Quantum Magnetism, Graduate Texts in Contemporary Physics (Spring-Verlag, Berlin, 1994).

${ }^{38}$ C. K. Majumdar and D. K. Ghosh, J. Math. Phys. 10, 1388 (1969).

${ }^{39}$ C. K. Majumdar and D. K. Ghosh, J. Math. Phys. 10, 1399 (1969).

${ }^{40}$ C. K. Majumdar, J. Phys. C 3, 911 (1970).

${ }^{41}$ Since we are interested in the long-range behavior of $C(l)$, the short distances were not considered in the fitting process. 